\section{(2) OPEN ACCESS}

\title{
Airborne occupational exposures and the risk of developing respiratory symptoms and airway obstruction in the Lifelines Cohort Study
}

\author{
Md Omar Faruque (D) , ${ }^{1,2}$ H Marike Boezen, ${ }^{1,2}$ Hans Kromhout, ${ }^{3}$ Roel Vermeulen, ${ }^{3}$ \\ Ute Bültmann, ${ }^{4}$ Judith M Vonk (D) ${ }^{1,2}$
}

- Additional material is published online only. To view please visit the journal online (http://dx.doi.org/10.1136/ thoraxjnl-2020-216721).

For numbered affiliations see end of article.

Correspondence to Professor H Marike Boezen, University Medical Center Groningen, Department of Epidemiology, University of Groningen, 9700 RB Groningen, Netherlands;

h.m.boezen@umcg.nl

Received 8 December 2020 Revised 27 January 2021 Accepted 5 February 2021

Published Online First 2 March 2021

\section{SLinked}

- http://dx.doi.org/10.1136/ thoraxjnl-2020-216626

Check for updates

(C) Author(s) (or their employer(s)) 2021. Re-use permitted under CC BY. Published by BMJ.

To cite: Faruque $\mathrm{MO}$,

Boezen HM, Kromhout $\mathrm{H}$, et al. Thorax

2021:76:790-797

\section{ABSTRACT}

Objectives To date, only a few studies have investigated the associations between occupational exposures and respiratory outcomes longitudinally in the general population. We investigated the associations between occupational exposures and the development of respiratory symptoms and airway obstruction in the Lifelines Cohort Study.

Methods We included 35739 occupationally active subjects with data on chronic cough, chronic phlegm, chronic bronchitis or airway obstruction at baseline and approximately 4.5 years follow-up. Exposures to biological dust, mineral dust, gases/fumes, pesticides, solvents and metals in the current job at baseline were estimated with the ALOHA+job-exposure matrix (JEM). Airway obstruction was defined as FEV, FVC below the lower limit of normal. Logistic regression analysis adjusted for baseline covariates was used to investigate the associations.

Results At follow-up, 1888 (6.0\%), 1495 (4.7\%), 710 (2.5\%) and 508 (4.5\%) subjects had developed chronic cough, chronic phlegm, chronic bronchitis and airway obstruction, respectively. High exposure to biological dust was associated with a higher odds to develop chronic cough and chronic bronchitis. High exposure to pesticides was associated with a higher odds for the development of all respiratory symptoms and airway obstruction. In the multiple exposures analyses, only the association between pesticides exposure and respiratory symptoms remained.

Conclusions Subjects exposed to high pesticides had a higher odds to develop respiratory symptoms on average 4.5 years later. Control measures should be taken to reduce pesticides exposure among the working population to prevent respiratory symptoms and airway obstruction.

\section{INTRODUCTION}

In the general population, the prevalence of respiratory symptoms for example, chronic bronchitis (presence of both chronic cough and phlegm) was estimated to be $0 \%-11 \% .^{1}$ Previous studies have reported that chronic bronchitis was associated with an accelerated lung function decline and a higher mortality rate. ${ }^{2-4}$ In 2017 , the global prevalence of COPD was estimated to be $3.9 \%$, and the disease accounts for 41.9 deaths per 100000 subjects which is $5.7 \%$ of total all-cause deaths. ${ }^{5}$ Set aside smoking, other factors such as occupational

\section{Key messages}

What is the key question?

- Are occupational exposures associated with the development of respiratory symptoms and airway obstruction in the general working population?

What is the bottom line?

- High occupational exposure to pesticides is associated with a higher odds to develop respiratory symptoms and airway obstruction in the general working population.

Why read on?

- We conducted this study in a large general working population who were followed for a median of 4.5 years and the occupational exposures were estimated with a job-exposure matrix.

exposures may also impair lung function by stimulating inflammatory responses on inhalation. ${ }^{6}$ Indeed, occupational exposures are responsible for $15 \%-20 \%$ of all COPD cases, ${ }^{7}$ with up to $31 \%$ in never smokers. ${ }^{8}$ Therefore, it is important to examine which occupational exposures are associated with the risk to develop respiratory symptoms and airway obstruction in the general population.

A Norwegian study showed an association between exposure to quartz, asbestos and dust/ fumes and the development of respiratory symptoms among subjects aged 15-70 years after 11 years of follow-up. ${ }^{9}$ After a follow-up of 20 years, the European Community Respiratory Health Survey (ECRHS) reported that exposure to mineral dust, gases/fumes and metals was associated with a higher risk to develop respiratory symptoms among subjects aged 20-44 years. ${ }^{10}$ Another study with the same population and after the same period of follow-up reported that occupational exposure to biological dust, gases/fumes and pesticides was associated with a 1.5-2.2-fold higher risk to develop airway obstruction. ${ }^{11}$ Consistently, after approximately 11 years of follow-up, a Swiss Cohort Study on Air Pollution and Lung and Heart Diseases in Adults reported that high exposure to biological dust, mineral dust, gases/fumes and vapours, gases, dusts or fumes was associated with a $1.5-4.5$-fold 


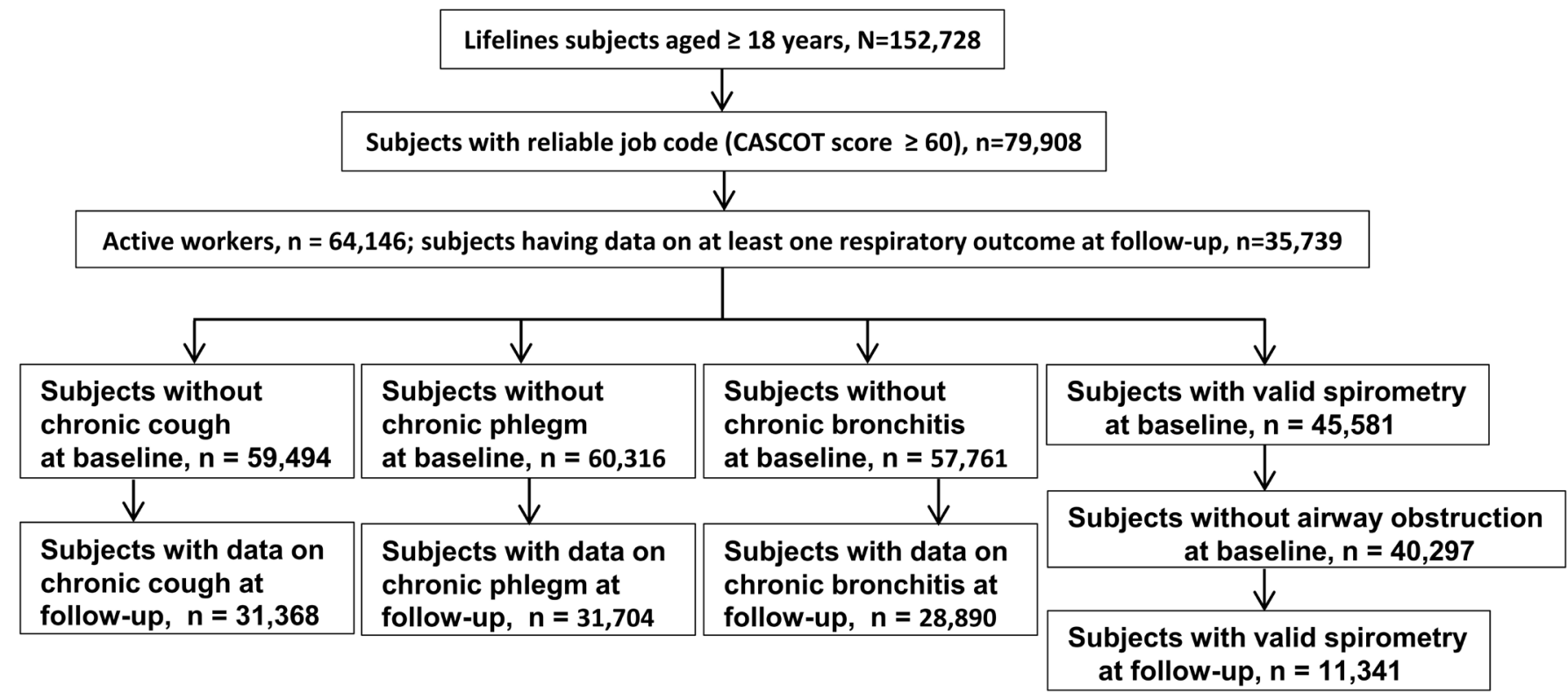

Figure 1 Flowchart of the selection of study subjects. CASCOT, Computer Assisted Structured Coding Tool.

higher risk to develop airway obstruction among subjects aged 18-62 years. ${ }^{12}$ Contrary, a recently published Danish nationwide register-based follow-up study showed an inverse association between exposure to biological dust and the development of airway obstruction among subjects aged $19-63$ years. ${ }^{13}$ The authors indicated that the lack of smoking data and a healthy worker survivor effect might have biased their results.

In the present study, we investigated the association between airborne exposure to biological dust, mineral dust, gases/fumes, pesticides, solvents and metals (estimated with a job-exposure matrix (JEM)) and the development of chronic cough, chronic phlegm, chronic bronchitis and airway obstruction in $>35000$ subjects from the Lifelines Cohort Study who were followed-up for 4.5 years. The Lifelines Cohort Study is a general populationbased study investigating subjects from the Northern part of the Netherlands. The strength of the Lifelines Cohort Study lies in the fact that its population is very homogeneous with respect to environmental exposures (eg, air pollution). Additionally, the consistency in regional and cultural work habits will reduce the variability in occupational exposures between people in the same job, compared with studies in which subjects from multiple countries were investigated (eg, the ECRHS).

\section{METHODS}

Population

In this study, we included 'occupationally active workers' from the Lifelines Cohort Study (figure 1). Baseline data were collected from 2006 to 2013 and the first follow-up visit was conducted between 2014 and 2017 after a median of 4.5 years (range: $1.8-8.8$ years). The scientific rationale, study design and survey methods of the Lifelines Cohort Study have been described elsewhere. ${ }^{14}$

\section{Occupational exposures}

We investigated the following occupational exposures: biological dust, mineral dust, gases/fumes, pesticides, solvents and metals. Occupational exposures were estimated using self-reported current job from the baseline questionnaire. The jobs were coded according to the International Standard Classification of
Occupations $^{15}$ using a Computer Assisted Structured Coding Tool (CASCOT). ${ }^{16}$ During this procedure, a CASCOT score was given to each coded occupation which indicates the probability that the given code is correct (range: 0-100). We selected subjects with a CASCOT score $\geq 60$, and all job titles above this score were reviewed and, if necessary, recoded to achieve accurate job coding. The ALOHA + JEM ${ }^{1718}$ was used to link occupational exposures (classified as no, low or high exposure) to the baseline jobs. For details, see online supplemental appendix 1 .

\section{Respiratory outcomes}

Chronic cough and chronic phlegm were self-reported both at baseline and follow-up, using the ECRHS questionnaire (for definitions, see online supplemental appendix 2). ${ }^{19}$ Chronic bronchitis was defined as the presence of both chronic cough and chronic phlegm. At baseline and follow-up, lung function was measured by prebronchodilator spirometry according to American Thoracic Society/European Respiratory Society (ATS/ERS) guidelines $^{20}$ using the Welch AllynSpiroPerfect device (Welch Allyn V.1.6.0.489, PC-based SpiroPerfect with CardioPerfect Workstation software). Airway obstruction was defined as the ratio of $\mathrm{FEV}_{1} / \mathrm{FVC}<$ lower limit of normal. ${ }^{21}$ Due to practical reasons, spirometry was performed in a random subset of the Lifelines participants.

\section{Covariates}

The subjects' age and sex were taken from the baseline screening. Education, monthly income, smoking status and pack-years were extracted from the baseline questionnaires.

\section{Statistical analyses}

Population characteristics were analysed for occupationally active subjects at baseline with data on at least one respiratory outcome at follow-up. In the current study, we used a follow-up design in which the exposure precedes the outcome, and thus only information about occupational exposures and covariates at baseline was included. To assess the correlation between occupational exposures, a non-parametric Spearman's rank-order 
correlation was used. To investigate the risk to develop chronic cough, we excluded subjects who reported chronic cough at baseline. Similarly, to investigate the risk to develop chronic sputum, chronic bronchitis and airway obstruction, we excluded subjects with chronic sputum, chronic bronchitis and airway obstruction, respectively, at baseline. Logistic regression was used to investigate the association between occupational exposures (no exposure to the specific agent as reference) and respiratory outcomes (chronic cough, chronic phlegm, chronic bronchitis and airway obstruction) at follow-up, adjusting for age, sex, education, monthly income, pack-years and smoking status (the type and categories of each covariate are given in table 1). All exposures were initially tested separately. Subsequently, we entered all exposures in one model to adjust for coexposures. A two-sided $\mathrm{p}$ value $<0.05$ was considered statistically significant.

\section{Sensitivity analyses}

To assess if the associations between occupational exposure and symptom development remain consistent if we use a more strict inclusion of asymptomatic subjects at baseline, we performed sensitivity analyses. We investigated the association between airborne occupational exposures and the development of respiratory symptoms including only subjects without both chronic cough and chronic phlegm at baseline. We additionally investigated each exposure in comparison to a common control group consisting of subjects with no exposure to any of the six occupational exposures under study. Finally, we investigated the association between occupational exposures and the development of respiratory symptoms and airway obstruction in subjects without asthma at baseline.

\section{RESULTS}

\section{Baseline characteristics}

Table 1 shows the baseline characteristics of the subjects having data on at least one respiratory outcome $(n=35739)$. At baseline, the mean age of the population was 43 years (SD: 10 years) and the majority was women (59.8\%). Approximately half of the subjects had received a medium education and a high monthly income. The median pack-years in ever smokers was 8 (IQR: 12) and about half of the subjects were ever smoker.

Exposure to gases/fumes was most prevalent $(45.7 \%$ with low or high exposure) followed by exposure to biological dust (34.3\%), and exposure to solvents (29.5\%) (table 1). Exposure to metals (4.9\%) and exposure to pesticides (5.2\%) were least prevalent. The prevalence of occupational exposures stratified by the respiratory outcomes is given in online supplemental table E1.

The Spearman rank correlation between baseline occupational exposures is shown in figure 2. The highest correlations were found between exposure to gases/fumes and biological dust $(r=0.54)$, mineral dust $(r=0.59)$ and solvents $(r=0.58)$. The weakest correlations were seen between exposure to pesticides and metals and solvents and between biological dust and metals.

\section{Occupational exposures and the development of respiratory symptoms and airway obstruction}

At follow-up, 1888 (6.0\%) subjects had developed chronic cough, 1495 (4.7\%) subjects had developed chronic phlegm, $710(2.5 \%)$ subjects had developed chronic bronchitis and 508 (4.5\%) subjects had developed airway obstruction.

In the adjusted models, high exposure to pesticides was associated with a 1.5-2.2-fold higher odds to develop respiratory symptoms and airway obstruction (table 2). In addition, high
Table 1 Population characteristics of symptom-free subjects at baseline

\begin{tabular}{|c|c|}
\hline \multicolumn{2}{|l|}{ Population characteristics, n=35 739} \\
\hline Age (years), mean (SD) & $43(10)$ \\
\hline Females (\%) & 59.8 \\
\hline \multicolumn{2}{|l|}{ Education } \\
\hline Low, n (\%) & $4365(12.3)$ \\
\hline Medium, n (\%) & $18467(52.2)$ \\
\hline High, n (\%) & $12016(34.0)$ \\
\hline Unclassifiable, n (\%) & $504(1.5)$ \\
\hline \multicolumn{2}{|l|}{ Monthly income } \\
\hline Low, n (\%) & $4231(12.0)$ \\
\hline Medium, n (\%) & $9416(26.6)$ \\
\hline High, n (\%) & $17189(48.8)$ \\
\hline Not reported, n (\%) & $4364(12.6)$ \\
\hline Pack-years in ever smokers, median (IQR) & $8(12)$ \\
\hline \multicolumn{2}{|l|}{ Smoking } \\
\hline Never smoker, n (\%) & $16979(48.8)$ \\
\hline Ex-smoker, n (\%) & $11259(32.4)$ \\
\hline Current smoker, n (\%) & $6541(18.8)$ \\
\hline $\mathrm{FEV}_{1} \%$ predicted, mean (SD) & $96.0(12.6)$ \\
\hline FVC\% predicted, mean (SD) & $100.1(12.0)$ \\
\hline $\mathrm{FEV}_{1} / \mathrm{FVC} \%$ predicted, mean (SD) & $95.4(7.8)$ \\
\hline \multicolumn{2}{|l|}{ Biological dust } \\
\hline No, n (\%) & $23252(65.7)$ \\
\hline Low, n (\%) & $10353(29.3)$ \\
\hline High, n (\%) & $1774(5.0)$ \\
\hline \multicolumn{2}{|l|}{ Mineral dust } \\
\hline No, $\mathrm{n}(\%)$ & $28094(79.4)$ \\
\hline Low, n (\%) & $5279(14.9)$ \\
\hline High, n (\%) & $2006(5.7)$ \\
\hline \multicolumn{2}{|l|}{ Gases/fumes } \\
\hline No, $n(\%)$ & $19228(54.3)$ \\
\hline Low, n (\%) & 13993 (39.6) \\
\hline High, n (\%) & $2158(6.1)$ \\
\hline \multicolumn{2}{|l|}{ Pesticides } \\
\hline No, n (\%) & $33553(94.8)$ \\
\hline Low, n (\%) & $1398(4.0)$ \\
\hline High, n (\%) & $428(1.2)$ \\
\hline \multicolumn{2}{|l|}{ Solvents } \\
\hline No, n (\%) & 24956 (70.5) \\
\hline Low, n (\%) & $8858(25.0)$ \\
\hline High, n (\%) & $1565(4.5)$ \\
\hline \multicolumn{2}{|l|}{ Metals } \\
\hline No, n (\%) & 33643 (95.1) \\
\hline Low, n (\%) & $1004(2.8)$ \\
\hline High, n (\%) & $732(2.1)$ \\
\hline
\end{tabular}

Education: low education (no training, primary education, lower or prevocational education); medium education (general secondary education, secondary vocational or professional guiding, preuniversity education); high education (higher professional or university degree); unclassifiable (subjects with other than above-mentioned education).
Monthly income: low-income (monthly net income $\leq € 1500$ ); medium-income (monthly net income between $€ 1500$ up and $€ 2500$ ); high-income (monthly net income $\geq € 2500$ ); unknown (I do not know/l do not want to say).
Smoking: never smokers (never smoked or smoked for $<1$ year); ex-smokers (smoked for $\geq 1$ year and stopped Smoking: never smokers (never smoked or smoked for $<1$ year); ex-smokers (smoked for
smoking for $\geq 1$ month); current smokers (current smoker or stopped smoking $<1$ month). smoking for $\geq 1$ month); current smokers (current smoker or stopped smok
$\mathrm{FEV}_{1}$, forced expiratory volume in 1 second; FVC, forced vital capacity.

exposure to biological dust was associated with a significant 1.3-1.5-fold higher odds to develop chronic cough and chronic bronchitis. In the model with all six airborne exposures, the odds estimates for high exposure to pesticides increased and remained 


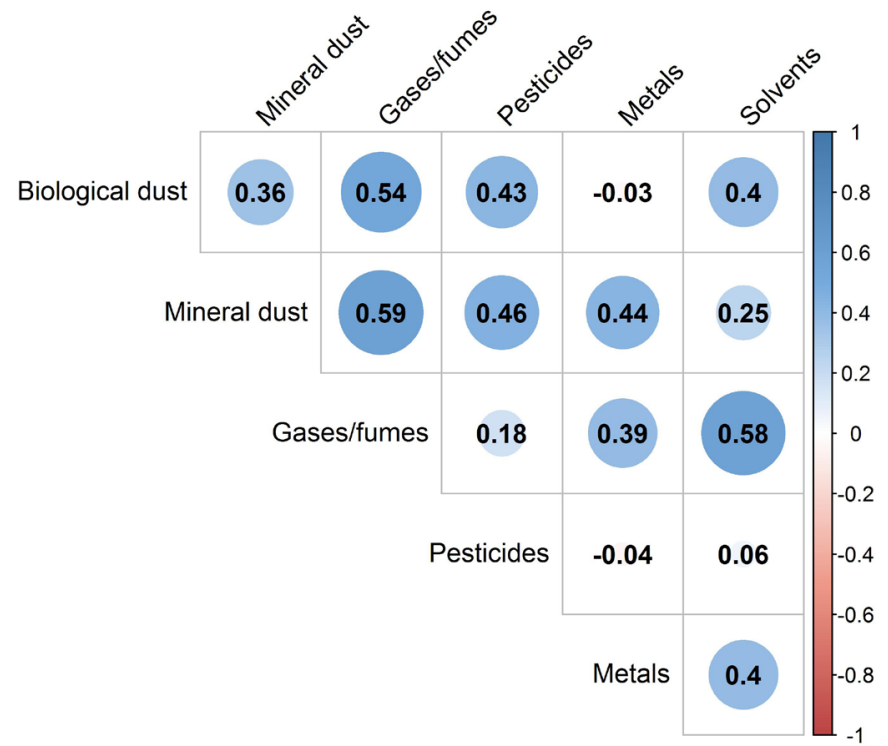

Figure 2 Correlogram shows the correlation among occupational exposures at baseline.

statistically significant for chronic phlegm and chronic bronchitis (table 3).

\section{Sensitivity analyses}

The associations between occupational exposures and the development of respiratory symptoms among subjects without both chronic cough and without chronic phlegm at baseline were comparable to the main findings (online supplemental table E2). In addition, the associations between occupational exposures and the development of respiratory symptoms and airway obstruction in comparison to a common control group consisting of subjects with no exposure to any of the six occupational agents were not notably different from the main findings (online supplemental table E3). The associations between occupational exposures and the development of respiratory symptoms and airway obstruction in subjects without asthma at the baseline were comparable to the main findings (online supplemental table E4).

\section{DISCUSSION}

Main findings

After a median follow-up of 4.5 years in the Lifelines Cohort Study, we found that subjects were at a higher odds to develop respiratory outcomes (chronic cough, chronic phlegm, chronic bronchitis and airway obstruction) on high occupational exposure to biological dust and pesticides. Mutual adjustment for the other exposures showed that only high pesticide exposure was persistently associated with the outcomes.

\section{Occupational exposures and the development of respiratory symptoms and airway obstruction}

We found that in the single exposure model, high occupational exposure to pesticides was associated with a higher odds to develop airway obstruction at follow-up. In line with our current findings, the ECRHS study found that in the single exposure model, exposure to pesticides was associated with a higher risk

Table 2 Associations between occupational exposures and the development of respiratory symptoms and airway obstruction

\begin{tabular}{|c|c|c|c|c|c|c|c|c|}
\hline \multirow{2}{*}{$\begin{array}{l}\text { Occupational } \\
\text { exposures }\end{array}$} & \multirow[b]{2}{*}{$N(31368)$} & \multirow{2}{*}{$\begin{array}{l}\text { Chronic cough } \\
\text { OR }(95 \% \mathrm{Cl})\end{array}$} & \multirow[b]{2}{*}{$N(31704)$} & \multirow{2}{*}{$\begin{array}{l}\text { Chronic phlegm } \\
\text { OR }(95 \% \mathrm{Cl})\end{array}$} & \multirow[b]{2}{*}{ N $(28890)$} & \multirow{2}{*}{$\begin{array}{l}\text { Chronic bronchitis } \\
\text { OR }(95 \% \mathrm{Cl})\end{array}$} & \multirow[b]{2}{*}{$N(11341)$} & \multirow{2}{*}{$\begin{array}{l}\text { Airway obstruction } \\
\text { OR }(95 \% \mathrm{Cl})\end{array}$} \\
\hline & & & & & & & & \\
\hline \multicolumn{9}{|l|}{ Biological dust } \\
\hline No & 20630 & Ref. & 20845 & Ref. & 18987 & Ref. & 7572 & Ref. \\
\hline Low & 9191 & 0.95 (0.85 to 1.06$)$ & 9317 & 0.93 (0.82 to 1.05$)$ & 8524 & 0.96 (0.80 to 1.15$)$ & 3226 & $1.17(0.95$ to 1.43$)$ \\
\hline High & 1547 & $1.26(1.03$ to 1.54$)$ & 1542 & 1.08 (0.86 to 1.36$)$ & 1379 & $1.46(1.07$ to 1.99$)$ & 543 & $1.17(0.75$ to 1.81$)$ \\
\hline \multicolumn{9}{|l|}{ Mineral dust } \\
\hline No & 25030 & Ref. & 25310 & Ref. & 23181 & Ref. & 9099 & Ref. \\
\hline Low & 4605 & 0.92 (0.80 to 1.07$)$ & 4664 & 1.01 (0.87 to 1.18 ) & 4193 & 1.02 (0.82 to 1.27$)$ & 1640 & $0.74(0.54$ to 1.00$)$ \\
\hline High & 1733 & 1.21 (1.00 to 1.46$)$ & 1730 & 1.08 (0.87 to 1.35$)$ & 1516 & 1.08 (0.78 to 1.49$)$ & 602 & $1.20(0.80$ to 1.79$)$ \\
\hline \multicolumn{9}{|l|}{ Gases/fumes } \\
\hline No & 17188 & Ref. & 17388 & Ref. & 15927 & Ref. & 6274 & Ref. \\
\hline Low & 12322 & 1.05 (0.94 to 1.17$)$ & 12452 & 0.99 (0.87 to 1.11$)$ & 11320 & 1.11 (0.93 to 1.32 ) & 4413 & 1.09 (0.89 to 1.33$)$ \\
\hline High & 1858 & $1.15(0.94$ to 1.40$)$ & 1864 & $1.04(0.83$ to 1.30$)$ & 1643 & 1.16 (0.84 to 1.59$)$ & 654 & $1.15(0.76$ to 1.74$)$ \\
\hline \multicolumn{9}{|l|}{ Pesticides } \\
\hline No & 29767 & & 30095 & Ref. & 27446 & Ref. & 10815 & Ref. \\
\hline Low & 1227 & $1.19(0.95$ to 1.50$)$ & 1232 & 1.03 (0.79 to 1.34$)$ & 1110 & $1.36(0.95$ to 1.93$)$ & 406 & 1.14 (0.69 to 1.87$)$ \\
\hline High & 374 & 1.45 (1.01 to 2.07$)$ & 377 & 1.49 (1.01 to 2.20$)$ & 334 & 1.99 (1.19 to 3.31$)$ & 120 & 2.24 (1.14 to 4.39$)$ \\
\hline \multicolumn{9}{|l|}{ Solvents } \\
\hline No & 22119 & Ref. & 22390 & Ref. & 20387 & Ref. & 8019 & Ref. \\
\hline Low & 7892 & 1.10 (0.98 to 1.23$)$ & 7961 & 1.01 (0.89 to 1.14 ) & 7292 & 1.12 (0.93 to 1.33$)$ & 2828 & 1.16 (0.94 to 1.43$)$ \\
\hline High & 1357 & 1.08 (0.86 to 1.34$)$ & 1353 & 1.06 (0.83 to 1.36$)$ & 1211 & 0.92 (0.64 to 1.34 ) & 494 & 1.03 (0.66 to 1.62$)$ \\
\hline \multicolumn{9}{|l|}{ Metals } \\
\hline No & 29877 & Ref. & 30216 & Ref. & 27574 & Ref. & 10784 & Ref. \\
\hline Low & 868 & 1.06 (0.81 to 1.37$)$ & 868 & 1.11 (0.83 to 1.48 ) & 775 & 1.21 (0.81 to 1.80$)$ & 338 & 0.72 (0.39 to 1.34$)$ \\
\hline High & 623 & $1.10(0.82$ to 1.48$)$ & 620 & $1.00(0.71$ to 1.41$)$ & 541 & $0.73(0.41$ to 1.29$)$ & 219 & $0.99(0.51$ to 1.92$)$ \\
\hline
\end{tabular}




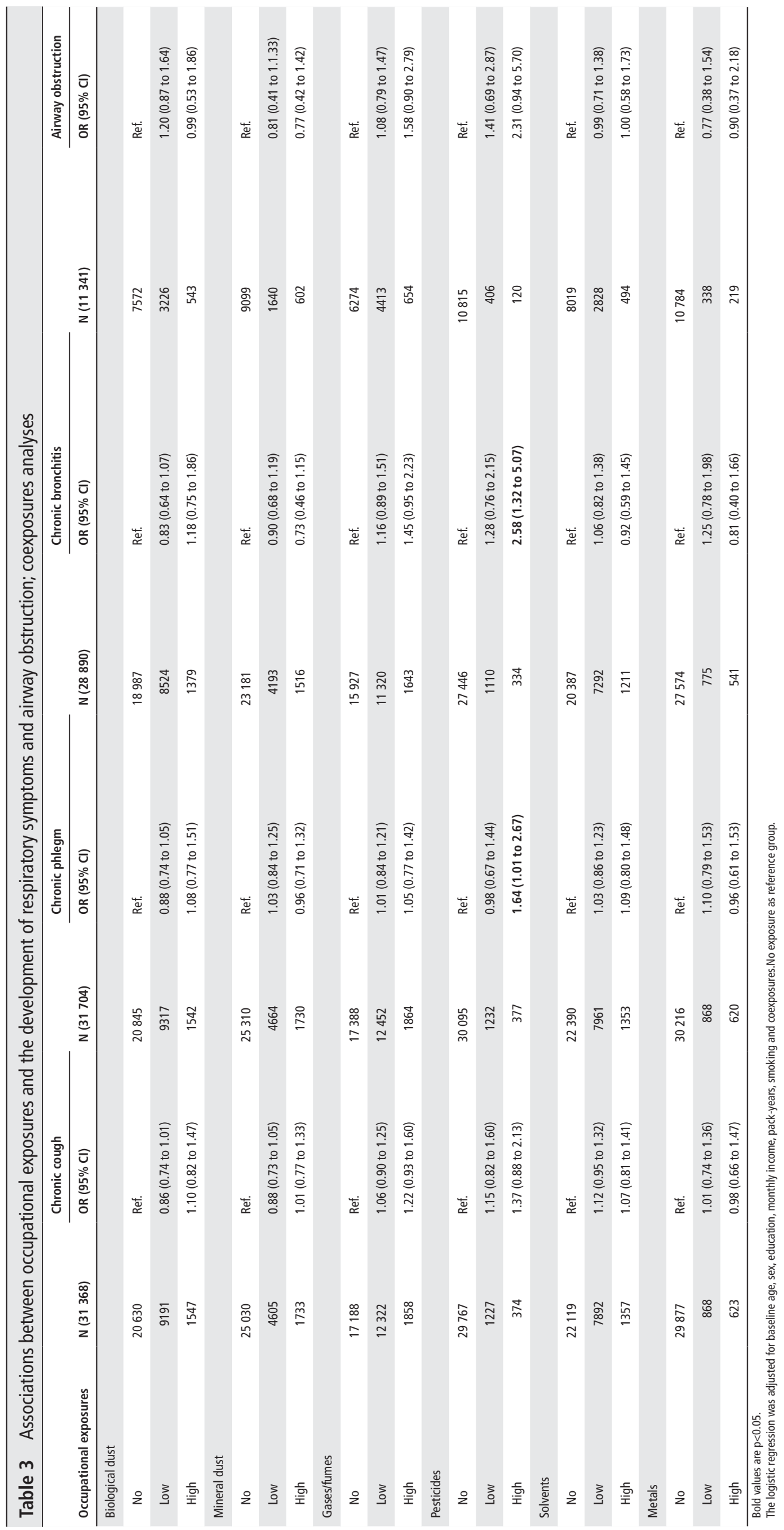

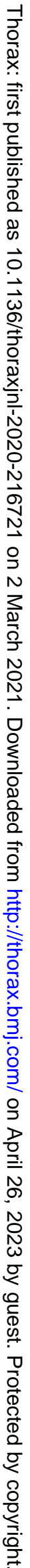


to develop airway obstruction after a follow-up of 20 years. ${ }^{11} \mathrm{~A}$ previous cross-sectional study within Lifelines also found that pesticides exposure was associated with a higher prevalence of airway obstruction. ${ }^{22}$ Our current findings strengthen the evidence that occupational exposure to pesticides is associated with a higher risk to develop airway obstruction in the general working population. Interestingly, in the coexposure model, the odds of the association between exposure to pesticides and airway obstruction increased (single exposure model vs coexposure model: 2.12 vs 2.23$)$, but became borderline $(p=0.091)$ significant.

Further, we found that high pesticides exposure is also a risk factor for developing respiratory symptoms. In contrast, the ECRHS study found no association between exposure to pesticides and the development of respiratory symptoms in the general population after 20 years of follow-up. ${ }^{10}$ Compared with the ECRHS study, in the current study, we included subjects with a wider age range (18-65 years vs $20-44$ years), but the follow-up period is much shorter than in the ECRHS study (4.5 years vs 20 years). In addition, we did not incorporate cumulative exposure in our exposure estimate as was done in the ECRHS study. Furthermore, large heterogeneity may exist in exposure to pesticides across the 30 centres of 15 European countries in the ECRHS study, whereas in our study all participants came from the three northern provinces of the Netherlands with considerably more farmers than in the more urbanised parts of the Netherlands. The participating ECRHS centres were also mainly urban centres and consequently, the number of farmers within the ECRHS study is relatively low. These discrepancies might explain the difference in the effect of pesticides exposure on respiratory health between the current study and the ECRHS study.

In the ECRHS study, metals exposure was associated with a higher risk to develop respiratory symptoms which was not the case in our study. The prevalence of metals exposure was higher in the ECRHS than in Lifelines $(\approx 11 \%$ vs $\approx 5 \%)$. There is not much heavy industry in the northern provinces of the Netherlands which could explain the low prevalence of metals exposure. In addition, in the Lifelines Cohort Study, women are over-represented $(\approx 60 \%)$, and not many females work in the metals industry.

The odds of developing respiratory symptoms and airway obstruction after being exposed to high pesticides remained significant in the analyses with adjustment for multiple exposures and even became somewhat stronger. This indicates that high exposure to pesticides at the workplace is the main driver of developing respiratory symptoms and airway obstruction among workers within Lifelines. Pesticides cover various chemical substances. To date, the biological mechanism through which the different active ingredients in pesticides affect the airways is poorly understood. A previous study reported that certain pesticides may induce inflammation by increasing neutrophil reactive oxygen molecule production and interleukin- 8 secretion. ${ }^{23}$ In addition, organophosphates and carbamates inhibit acetylcholinesterase, which results in mucus hypersecretion and airway smooth muscle contraction. ${ }^{24}$ Thus, persistent inflammation induced by pesticides might result in chronic respiratory symptoms and airway obstruction.

Previously, we found that occupational exposure to pesticides was associated with a lower level of inflammatory biomarkers (C reactive protein and neutrophils), and was not associated with changes in biomarkers after 4.5 years follow-up. ${ }^{25}$ This finding indicates that pesticides are not leading to higher levels of inflammation and may thus alter or damage our immune system through other biological pathways. Cytokines pathways, induction of oxidative stress, mitochondrial dysfunction, endoplasmic reticulum stress, disruption of the ubiquitin protease system or autophagy, inhibition of enzymes with esterase activity, and endocrine disruption are some suggested biological pathways through which pesticides could disrupt the immune system. ${ }^{26}{ }^{27}$ Further research is required to shed light on the respiratory health risks of specific active ingredients of pesticides, the biological mechanism, and the exposure-response relationship.

In the analyses without adjustment for coexposure, we found that symptom-free individuals with high biological dust exposure had a higher odds of developing chronic cough and chronic bronchitis. These significant associations between high biological dust exposure and the development of symptoms disappeared in the analyses with all exposures and only the association between high pesticides exposure and symptom development remained. We observed that all pesticide exposed workers, for example, crop growers $(n \approx 110)$, gardeners $(n \approx 110)$, animal producers $(n \approx 530)$ and labours in agriculture and forest $(n \approx 5)$, were also exposed to biological dust, but not all biological dust exposed workers were exposed to pesticides (eg, fibre preparers, weavers, knitters and paper-making plants operators). We examined the association between exposure to biological dust and the development of respiratory symptoms among these subjects who were exposed only to biological dust but not to pesticides. The analyses showed no significant association between biological dust exposure and the development of respiratory symptoms (online supplemental table E5). This indicates that the significant association between exposure to biological dust and respiratory symptoms was confounded by exposure to pesticides.

In the general population-based Vlagtwedde-Vlaardingen study, we showed that pesticides exposure was associated with accelerated lung function decline after 25 years of follow-up in the 70 s. $^{28}$ In 1979, the guidelines on pesticides were first legislated at the European Union level, and have evolved considerably over the years. ${ }^{29}$ Thus, our current study findings indicate that the existing policies and legislation on pesticides may still not be adequate to protect the workers from the adverse respiratory health effects of occupational exposure to pesticides. Recent studies conducted in low-income and middle-income countries also showed that farmers with pesticides exposure were at a higher risk of developing respiratory symptoms and airway obstruction. ${ }^{30-32}$

\section{Strengths and limitations}

In this study, we included a very large number of occupationally active subjects covering a wide age range and followed for a median of 4.5 years from the Lifelines Cohort Study. Lifelines contains a large amount of quality data which allowed us to adjust for important confounders. Our study population is homogenous in terms of ethnicity, geographical locations and other environmental exposures such as air pollution, and therefore, our study findings are not confounded by these factors. The participants of the Lifelines Cohort Study are representative of the general population of the three northern provinces of the Netherlands. ${ }^{33}$ In addition, data on respiratory symptoms were measured with a validated questionnaire and lung function was measured according to a standardised protocol. We performed additional analyses to assess the effect of coexposure in our findings.

We used the expert-based ALOHA+JEM to estimate occupational exposure. We prefer the use of a JEM over selfreported exposure since workers often struggle to recall detailed 
information on working conditions many years back, and in many instances, they link their disease condition with previous exposure (recall bias). An objectively constructed JEM is a more robust tool in estimating occupational exposure and eliminating differential bias. ${ }^{34}$ A JEM by definition does not account for differences in exposure levels observed between individuals with the same reported job. ${ }^{35}$ However, since our study population is from the same geographical region, the regional and cultural work habits will minimise this variability in occupational exposures between individuals with the same job. The ALOHA+JEM also does not assess exposure at the individual chemical or biological level. All these shortcomings may lead to imprecision, but due to the Berkson nature of this error, the presented odds estimates will be hardly or not biased. ${ }^{36}$ In this study, we used the information about occupational exposure at baseline and did not take into account potential changes in occupational exposures between baseline and follow-up. Given that the duration of follow-up is relatively short (median 4.5 years), we do not expect that many people have changed their jobs, however, we cannot entirely rule out the possible impact of these changes on the outcomes. In addition, the findings of this study are based on a homogenous population from the northern Netherlands, which may limit the generalisability to other populations to some extent.

\section{CONCLUSION}

In this study, we found that high occupational exposure to pesticides was associated with a higher odds of developing respiratory symptoms and airway obstruction among the general working population. More rigorous efforts are required to protect workers from the adverse health effects of occupational pesticides exposure. This can be done by adopting a hierarchy of pesticide control measures, starting from a reduction or elimination of pesticides in the workplace to substitution by alternative materials and to the engineering of control measures (eg, enclosure or isolation of the hazardous work process) and administrative measures (eg, routine surveillance of the safety management system and guidelines).

\section{Author affiliations \\ ${ }^{1}$ University Medical Center Groningen, Department of Epidemiology, University of Groningen, Groningen, Netherlands \\ ${ }^{2}$ University Medical Center Groningen, Groningen Research Institute for Asthma and COPD (GRIAC), University of Groningen, Groningen, Netherlands \\ ${ }^{3}$ Institute for Risk Assessment Sciences (IRAS), Division of Environmental Epidemiology, Utrecht University, Utrecht, Netherlands \\ ${ }^{4}$ University Medical Center Groningen, Department of Health Sciences, Community and Occupational Medicine, University of Groningen, Groningen, Netherlands}

Contributors All authors contributed to the conception, design and interpretation of the results. MOF analysed data, produced tables and graphs, and wrote the first version of the manuscript. JMV, HMB and UB critically reviewed the manuscript, tables and graphs, and supplementary materials. HK and RV developed the ALOHA+JEM and carefully reviewed the manuscript. All authors approved the final version of the manuscript.

Funding MOF was supported by the PhD Scholarship programme of the Graduate School of Medical Sciences (GSMS), University of Groningen. The Lifelines Cohort Study was supported by the Dutch Ministry of Health, Welfare and Sport, the Ministry of Economic Affairs, Agriculture and Innovation, the Province of Groningen, the European Union (Regional Development Fund), the Northern Netherlands Provinces (SNN), the Netherlands Organisation for Scientific Research (NWO), the University Medical Center Groningen (UMCG), the University of Groningen, de Nierstichting (the Dutch Kidney Foundation) and the Diabetes Fonds (the Diabetic Foundation). The sponsors of this study had no role in the design of this study, data collection, analysis and interpretation or in writing and submitting of the manuscript.

Competing interests None declared.

Patient consent for publication Not required.
Ethics approval This study was approved by the Medical Ethical Commission (METC) of the University Medical Center Groningen (Reference number-2007/152). All subjects signed written informed consent and all methods were carried out in accordance with relevant guidelines and regulations for human subjects.

Provenance and peer review Not commissioned; externally peer reviewed.

Data availability statement Data may be obtained from a third party and are not publicly available. Registration is required to obtain data from the Lifelines Cohort Study. It is not permitted to deposit the Lifelines data in an open data repository. To obtain data, used in the current study, interested researchers should contact the Lifelines Cohort Study (www.lifelines.nl).

Open access This is an open access article distributed in accordance with the Creative Commons Attribution 4.0 Unported (CC BY 4.0) license, which permits others to copy, redistribute, remix, transform and build upon this work for any purpose, provided the original work is properly cited, a link to the licence is given, and indication of whether changes were made. See: https://creativecommons.org/ licenses/by/4.0/.

\section{ORCID iDs}

Md Omar Faruque http://orcid.org/0000-0003-3806-6606

Judith M Vonk http://orcid.org/0000-0001-7531-4547

\section{REFERENCES}

1 Mejza F, Gnatiuc L, Buist AS, et al. Prevalence and burden of chronic bronchitis symptoms: results from the BOLD study. Eur Respir J 2017;50:1700621.

2 Guerra S, Sherrill DL, Venker C, et al. Chronic bronchitis before age 50 years predicts incident airflow limitation and mortality risk. Thorax 2009;64:894-900.

3 Stavem K, Sandvik L, Erikssen J. Can global initiative for chronic obstructive lung disease stage 0 provide prognostic information on long-term mortality in men? Chest 2006;130:318-25.

4 Ekberg-Aronsson M, Pehrsson K, Nilsson J-A, et al. Mortality in gold stages of COPD and its dependence on symptoms of chronic bronchitis. Respir Res 2005;6:98.

5 GBD Chronic Respiratory Disease Collaborators. Prevalence and attributable health burden of chronic respiratory diseases, 1990-2017: a systematic analysis for the global burden of disease study 2017. Lancet Respir Med 2020;8:585-96.

6 Prince $\mathrm{P}$, Lemière $\mathrm{C}$, Dufour $\mathrm{M}-\mathrm{H}$, et al. Airway inflammatory responses following exposure to occupational agents. Chest 2012;141:1522-7.

7 Society ER, Society ER. European lung Foundation. occupational lung diseases. . Eur Lung White B 2013:282-95.

8 Hnizdo E, Sullivan PA, Bang KM, et al. Association between chronic obstructive pulmonary disease and employment by industry and occupation in the US population: a study of data from the third National health and nutrition examination survey. Am J Epidemiol 2002; 156:738-46.

9 Eagan TML, Gulsvik A, Eide GE, et al. Occupational airborne exposure and the incidence of respiratory symptoms and asthma. Am J Respir Crit Care Med 2002;166:933-8.

10 Lytras T, Kogevinas M, Kromhout $H$, et al. Occupational exposures and incidence of chronic bronchitis and related symptoms over two decades: the European community respiratory health survey. Occup Environ Med 2019;76:222-9.

11 Lytras T, Kogevinas M, Kromhout H, et al. Occupational exposures and 20-year incidence of COPD: the European community respiratory health survey. Thorax 2018;73:1008-15.

12 Mehta AJ, Miedinger D, Keidel D, et al. Occupational exposure to dusts, gases, and fumes and incidence of chronic obstructive pulmonary disease in the Swiss cohort study on air pollution and lung and heart diseases in adults. Am J Respir Crit Care Med 2012:185:1292-300.

13 Vested A, Basinas I, Burdorf A, et al. A nationwide follow-up study of occupational organic dust exposure and risk of chronic obstructive pulmonary disease (COPD). Occup Environ Med 2019;76:105-13.

14 Scholtens S, Smidt N, Swertz MA, et al. Cohort profile: lifelines, a three-generation cohort study and Biobank. Int J Epidemiol 2015;44:1172-80.

15 International Labour Organization. International standard classification of occupations 2008 (ISCO-08), 2016. Available: http://www.ilo.org/public/english/bureau/stat/isco-/ isco08/index.htm [Accessed 15 Aug 2020].

16 Jones R, Elias P. CASCOT: computer-assisted structured coding tool. Coventry, UK: Institute for Employment Research, University of Warwick, 2004.

17 Sunyer J, Zock JP, Kromhout $\mathrm{H}$, et al. Lung function decline, chronic bronchitis, and occupational exposures in young adults. Am J Respir Crit Care Med 2005:172:1139-45.

18 Sunyer J, Kogevinas M, Kromhout $\mathrm{H}$, et al. Pulmonary ventilatory defects and occupational exposures in a population-based study in Spain. Spanish group of the European community respiratory health survey. Am J Respir Crit Care Med 1998;157:512-7.

19 Heinrich J, Richter K, Frye C. Die Europäische Studie zu Atemwegserkrankungen bei Erwachsenen (ECRHS) - Bisherige Ergebnisse und der Beitrag der beiden deutschen Studienzentren [European Community Respiratory Health Survey in Adults (ECRHS)] Pneumologie 2002;56:297-303. 
20 Miller MR, Hankinson J, Brusasco V, et al. Standardisation of spirometry. Eur Respir J 2005:26:319-38.

21 Stanojevic S, Wade A, Stocks J, et al. Reference ranges for spirometry across all ages: a new approach. Am J Respir Crit Care Med 2008;177:253-60.

22 de Jong K, Boezen HM, Kromhout $\mathrm{H}$, et al. Pesticides and other occupational exposures are associated with airway obstruction: the lifelines cohort study. Occup Environ Med 2014;71:88-96.

23 Pelletier M, Roberge CJ, Gauthier M, et al. Activation of human neutrophils in vitro and dieldrin-induced neutrophilic inflammation in vivo. J Leukoc Biol 2001;70:367-73.

24 Doust E, Ayres JG, Devereux G, et al. Is pesticide exposure a cause of obstructive airways disease? Eur Respir Rev 2014;23:180-92.

25 Faruque MO, Vonk JM, Bültmann U, et al. Airborne occupational exposures and inflammatory biomarkers in the lifelines cohort study. Occup Environ Med 2021;78:93

26 Gangemi S, Gofita E, Costa C, et al. Occupational and environmental exposure to pesticides and cytokine pathways in chronic diseases (review). Int J Mol Med 2016;38:1012-20.

27 Mokarizadeh A, Faryabi MR, Rezvanfar MA, et al. A comprehensive review of pesticides and the immune dysregulation: mechanisms, evidence and consequences. Toxicol Mech Methods 2015;25:258-78.

28 de Jong K, Boezen HM, Kromhout H, et al. Association of occupational pesticide exposure with accelerated longitudinal decline in lung function. Am J Epidemiol 2014; 179:1323-30.
29 Skevas T, Oude Lansink AGJM, Stefanou SE. Designing the emerging EU pesticide policy: a literature review. NJAS - Wageningen Journal of Life Sciences 2013;6465:95-103.

30 Negatu B, Kromhout $H$, Mekonnen Y, et al. Occupational pesticide exposure and respiratory health: a large-scale cross-sectional study in three commercial farming systems in Ethiopia. Thorax 2017;72:522-9.

31 Schenker MB, Stoecklin M, Lee K, et al. Pulmonary function and exercise-associated changes with chronic low-level paraquat exposure. Am J Respir Crit Care Med 2004;170:773-9.

32 Chakraborty S, Mukherjee S, Roychoudhury S, et al. Chronic exposures to cholinesterase-inhibiting pesticides adversely affect respiratory health of agricultural workers in India. J Occup Health 2009;51:488-97.

33 Klijs B, Scholtens S, Mandemakers JJ, et al. Representativeness of the lifelines cohort study. PLoS One 2015;10:e0137203.

34 Kauppinen TP, Mutanen PO, Seitsamo JT. Magnitude of misclassification bias when using a job-exposure matrix. Scand J Work Environ Health 1992;18:105-12.

35 Kromhout H, Symanski E, Rappaport SM. A comprehensive evaluation of within- and between-worker components of occupational exposure to chemical agents. Ann Occup Hyg 1993;37:253-370.

36 Armstrong BG. Effect of measurement error on epidemiological studies of environmental and occupational exposures. Occup Environ Med 1998;55:651-6. 